\title{
Discrimination of intertrial intervals in cross-modal transfer of duration
}

\author{
WARREN H. MECK and RUSSELL M. CHURCH \\ Brown University, Providence, Rhode Island 02912
}

\begin{abstract}
Twenty rats were trained to press one lever ("short") following a 2-sec light signal and the other lever ("long") following an 8-sec light signal. The interval from the end of one trial to the onset of the signal on the next trial was $30 \mathrm{sec}$. Half the animals were tested on a discrimination between 2-sec and 8-sec noise signals with the same response rule (nonreversal); half were tested with the opposite response rule (reversal). On cross-modal testing, the nonreversal group was correct more often than chance; the reversal group was correct less often than chance. When signals were omitted, the animals performed better than chance, indicating that they discriminated the difference in time from the end of one trial until the opportunity for a choice on the next trial $(32 \mathrm{vs} .38 \mathrm{sec})$.
\end{abstract}

A discrete-trials choice procedure was used to measure any cross-modal transfer of specific learning between vision and audition. The rats were first trained to classify the duration of light signals as short (left response) or long (right response) with a procedure that leads to rapid temporal discrimination learning (e.g., Church \& Deluty, 1977; Maricq, Roberts, \& Church, 1981). Following this training with light signals, animals were retrained with noise signals. To obtain a sensitive indication of cross-modal transfer, half of the animals were given nonreversal cross-modal transfer (i.e., reinforcement of a left response following a short noise signal and reinforcement of a right response following a long noise signal) and half of the animals were given reversal cross-modal retraining (i.e., reinforcement of a left response following a long noise signal and reinforcement of a right response following a short noise signal). This design has been used by others (e.g., Over \& Mackintosh, 1969; Wilson \& Shaffer, 1963). If there is cross-modal transfer of the attribute of duration from light to noise, the percentage of correct responses should be greater in the nonreversal than the reversal group.

\section{METHOD}

\section{Subjects and Apparatus}

The subjects were 20 experimentally naive male albino Norway rats (Charles River CD), about 10 months old when the experiment began. Animals were individually housed in metal cages in which water was continuously available. Immediately after the daily session, the rats were fed a daily ration of about $14 \mathrm{~g}$ of Charles River Rat Formula mixed with water. A lightdark cycle of $16 \mathrm{~h}$ light and $8 \mathrm{~h}$ dark was maintained in the animal colony, with light onset at 7:00 a.m.

The rats worked in 10 standard lever boxes described elsewhere (Meck \& Church, in press). A timeshared PDP-12 computer con-

This research was supported by National Science Foundation (NSF) Research Grant BNS 79-04792. Requests for reprints should be sent to Warren H. Meck, Department of Psychology, Brown University, Providence, Rhode Island 02912. trolled the experimental equipment and recorded the responses.

\section{Procedure}

Pretraining. Each rat received at least two sessions of continuous reinforcement training as described elsewhere (Meck \& Church, in press).

Training (Days 1-10). The rats were trained to press the left lever (short response) following a light termination signal of $2 \mathrm{sec}$ duration and to press the right lever (long response) following a signal of $8 \mathrm{sec}$ duration. On each trial, one of the two signal durations was randomly presented with a probability of .5 . The houselight went off for the selected duration; at the end of this period of time, the houselight was turned back on and both levers were inserted into the box. If the animal made the correct response, a pellet of food was immediately delivered; if it made the incorrect response, no pellet was delivered. When either lever was pressed, there was a $.5 \mathrm{sec}$ delay, and then both levers retracted. The intertrial interval was defined as the time from the end of one trial until the onset of the signal on the next trial. After a fixed $30 \mathrm{sec}$ intertrial interval, another trial began. On Days 1-5, if an incorrect response had been made on the previous trial, the same signal duration occurred again on the next trial (correction procedure). From Day 6 until the end of the experiment, there were no correction trials. Daily sessions began at noon and 2:00 p.m. and lasted $1 \mathrm{~h}$ and $50 \mathrm{~min}$.

Cross-modal training (Days 11-20). The first 20 trials of the 1 st day of cross-modal training consisted of $2-\mathrm{sec}$ or $8-\mathrm{sec}$ houselight termination signals (i.e., the same procedure used on Days 6-10). No data were collected on these trials. Thereafter, rats were retrained with a $2-\mathrm{sec}$ or $8-\mathrm{sec}$ white noise signal. Half the rats were tested with the same response rule (reinforcement of a left response following a $2 \mathrm{sec}$ signal and reinforcement of a right response following an 8-sec signal), and half the rats were tested with a reversed response rule (reinforcement of a right response following an 8 -sec signal and reinforcement of a left response following a 2 -sec signal).

Signal omission training (Days 21-30). The procedure was the same as that used in cross-modal training, except the signal was omitted. Thus, there was either a $32 \mathrm{sec}$ or a $38 \mathrm{sec}$ interval between the end of one trial and the opportunity for a choice response on the next trial. When this interval was $32 \mathrm{sec}$, a short response was reinforced; when it was $38 \mathrm{sec}$, a long response was reinforced.

Retraining with a fixed intertrial interval (Days 31-35). The procedure was identical to that used in the cross-modal training phase. 
Retraining with a random intertrial interval (Days 36-40). The procedure was the same as that used in retraining, except that the intertrial interval was now $5 \mathrm{sec}$ plus a geometrically distributed duration with a minimum of $.1 \mathrm{sec}$ and a mean of $40 \mathrm{sec}$.

\section{RESULTS}

The performance of the nonreversal and reversal groups was similar throughout training. During the last 5 days of training, the mean percentage of correct responses of rats in the nonreversal group $(86 \% \pm 2 \%)$ was not significantly different from the mean percentage of correct responses of rats in the reversal group $(86 \% \pm 3 \%)$. (The values in parentheses refer to the mean percent and 1 standard error of the mean.)

Figure 1 shows the percentage correct for individual subjects in each group averaged over responses to both 2 -sec and 8-sec durations on the 1st day of cross-modal training. As shown in the left side of the figure, the nonreversal group had a mean of $73 \% \pm 3 \%$ correct, significantly greater than chance $[\mathrm{t}(9)=8.8, \mathrm{p}<.001]$. The transfer, however, was not perfect, since this was significantly lower than the mean percent correct during the previous five sessions $[t(9)=5.0, p<.001]$. On the same 1st day of cross-modal training, as shown in the right side of Figure 1, the reversal group had a mean of $42 \% \pm 3 \%$ correct, significantly less than the nonreversal

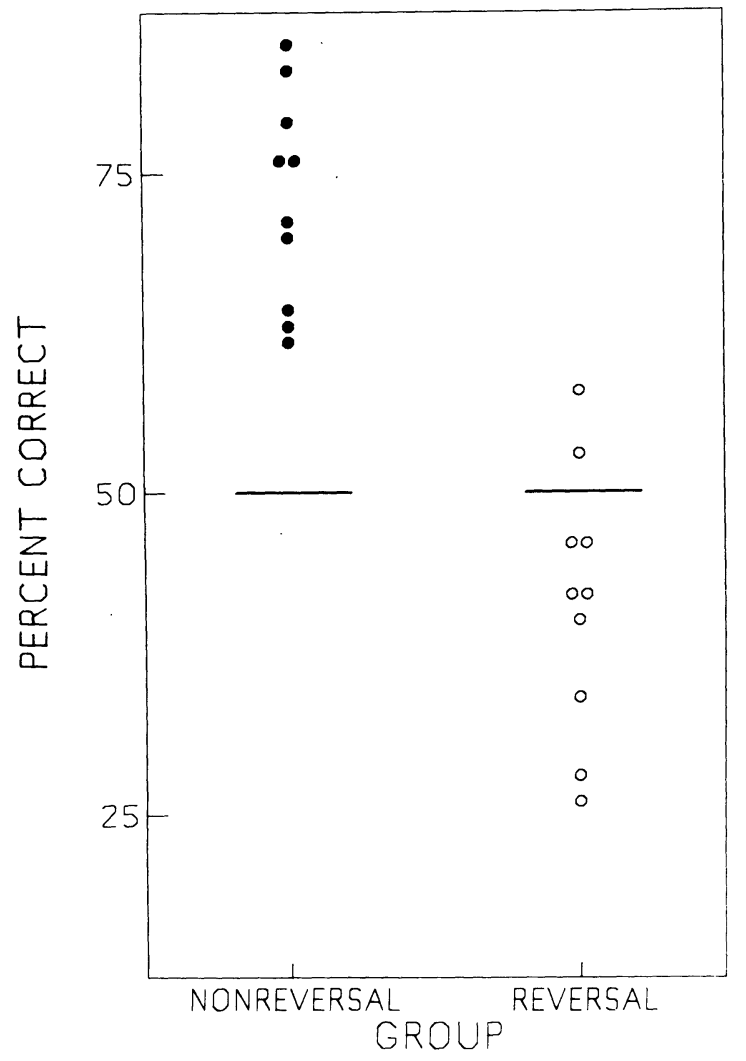

Figure 1. Percent correct on the 1st day of cross-modal transfer training for 10 rats in the nonreversal group and 10 rats in the reversal group.

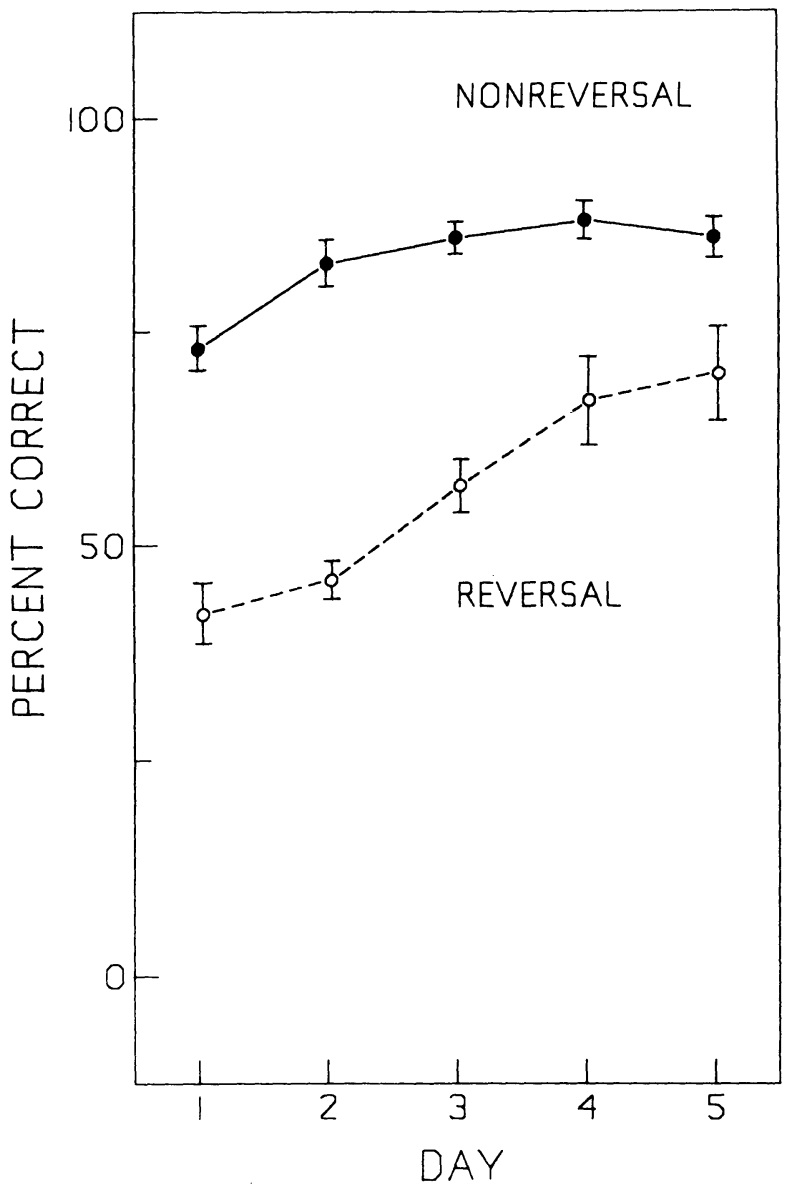

Figure 2. Mean percent correct as a function of day of crossmodal transfer for the nonreversal and reversal groups. The vertical lines indicate \pm 1 standard error of the mean.

group $[t(18)=7.42, p<.001]$ and significantly less than chance $[t(9)=2.57, \mathrm{p}<.05]$.

The results of the first 50 trials of cross-modal transfer were even more striking. The nonreversal group was correct on $71 \% \pm 2 \%$ of the trials, and all 10 rats in this group were correct on more than $50 \%$ of the trials; the reversal group was correct on only $33 \% \pm 3 \%$ of the trials, and all 10 rats were correct on less than $50 \%$ of the trials.

Figure 2 shows that the difference between groups was maintained during the first 5 days of retraining $[\operatorname{ts}(18) \geqslant 3.05$, ps $<.01]$. (The vertical distances are +1 and -1 standard error of the mean.)

Table 1 shows that on the 1st day of signal omission training, rats in both the nonreversal group and the reversal group responded correctly more often than chance $[\operatorname{ts}(9) \geqslant 2.15$, ps $<.05]$. The nonreversal group responded correctly more often than the reversal group $[\mathrm{t}(18)=2.12, \mathrm{p}<.05]$. Performance during signal omission training was poorer than performance during the 1st day of cross-modal training with sound signals for both groups $[\operatorname{ts}(9) \geqslant 2.5$, ps $<.05]$. The mean percent correct for all rats during the last 5 days of crossmodal training on the $2-\mathrm{sec}$ vs. $8-\mathrm{sec}$ discrimination was 
Table 1

Percentage Correct During the 1st Day of Signal Omission Training

\begin{tabular}{ccc} 
& \multicolumn{2}{c}{ Group } \\
\cline { 3 - 3 } Rat & Nonreversal & Reversal \\
\hline 1 & 61 & 55 \\
2 & 62 & 56 \\
3 & 64 & 50 \\
4 & 63 & 52 \\
5 & 56 & 47 \\
6 & 53 & 52 \\
7 & 63 & 58 \\
8 & 49 & 52 \\
9 & 66 & 52 \\
10 & 60 & 69 \\
Mean & 60 & 54 \\
SE & 2 & 2 \\
\hline
\end{tabular}

$81 \% \pm 2 \%$, whereas the mean percent correct for the last 5 days of cross-modal training on the 32-sec vs. 38 -sec discrimination was $63 \% \pm .4 \%$ correct, a reliable difference between the two conditions $[t(9)=9.5$, $\mathrm{p}<.001]$.

The mean percentage correct during the 5 days of retraining with a fixed intertrial interval was $86 \% \pm 3 \%$. On the 1st day of training with random intertrial intervals, the mean percent correct on the 2 -sec vs. 8 -sec discrimination was $88 \% \pm 3 \%$, a nonsignificant change from retraining $[t(9)=1.5, p \geqslant .05]$. The mean percentage correct for animals with random intertrial intervals during the 5 days of retraining was $87 \% \pm 2 \%$.

\section{DISCUSSION}

The purpose of this experiment was to demonstrate positive and negative cross-modal transfer from light to sound of a specific duration discrimination. The results were similar to others reported from our laboratory (e.g., Meck \& Church, in press; Roberts, 1982). The amount of transfer was greater, and it lasted longer than the cross-modal transfer by animals previously reported (e.g., Over \& Mackintosh, 1969).

When the signal was omitted, the rats were still able to perform better than chance. How was this possible? The determination of trial condition was random with replacement, so that the animal could not correctly anticipate any regularities in the trial sequence. The experiment, however, did use a fixed intertrial interval of $30 \mathrm{sec}$. From the signal omission training, it became clear that the animals were able to select a correct response not only on the basis of the difference in signal duration ( 2 vs. $8 \mathrm{sec}$ ), but also on the basis of the interval between the end of one trial and the insertion of the levers $(32 \mathrm{vs} .38 \mathrm{sec})$. When the signals were no longer presented, the animals used the other temporal cue of the intertrial interval. The accuracy of performance was lower during signal omission training because of the scalar property of time perception (e.g., Gibbon, 1977).

Why the animals learned the relationship concerning the interval between the end of one trial and the insertion of the levers on the next trial is uncertain, since signal duration provided the basis for more accurate performance. Apparently, during training, they learned the relationship between response and reinforcement as a function of both signal duration and the interval from the end of one trial to the opportunity for a response. Both intervals could be used simultaneously to predict which response will be reinforced (Meck \& Church, Note 1). Such redundant relevant stimuli can improve learning and performance (e.g., Mackintosh, 1974, pp. 570-579). Alternatively, the intertrial interval may have alerted the animal to impending signal onset. This would have led to the preparatory response of directing attention to the relevant channel of sensory input, thus facilitating information processing (e.g., Posner, 1978). Despite the discrimination of intertrial intervals, there are two lines of evidence for cross-modal transfer in this experiment. First, there was no difference in discrimination of signal duration between fixed and variable intertrial interval conditions, so the fixed intertrial interval was not essential for the discrimination of signal duration. Second, performance on the 1st day of cross-modal transfer was substantially better than during signal omission training. The magnitude of cross-modal transfer is uncertain because of the confounding effect of the interval between the end of one trial and the opportunity for a response on the next trial.

In a duration-discrimination experiment, if the time from the end of one trial to the onset of a signal is held constant, the animal may use the time from the end of one trial until the opportunity for a response on the next trial as a discriminative stimulus. A random intertrial interval does not completely eliminate this problem, since the discrimination can be based on the central tendency of the two distributions of intervals between the end of one trial and the opportunity for response on the next trial. A random intertrial interval with a large variance relative to the mean, however, can reduce the problem to a negligible amount.

\section{REFERENCE NOTE}

1. Meck, W. H., \& Church, R. M. Simultaneous temporal processing. Manuscript in preparation, 1982.

\section{REFERENCES}

Church, R. M., Deluty, M. Z. Bisection of temporal intervals. Journal of Experimental Psychology: Animal Behavior Processes, 1977, 3, 216-228.

GibBon, J. Scalar expectancy theory and Weber's law in animal timing. Psychological Review, 1977, 84, 279-325.

Mackintosh, N. J. The psychology of animal learning. New York: Academic Press, 1974.

MaricQ, A. V., Roberts, S., \& Church, R. M. Methamphetamine and time estimation. Journal of Experimental Psychology: Animal Behavior Processes, 1981, 7, 18-30.

Meck, W. H., \& ChURCh, R. M. Abstraction of temporal attributes. Journal of Experimental Psychology: Animal Behavior Processes, in press.

Over, R., \& MaCkintosh, N. J. Cross-modal transfer of intensity discrimination by rats. Nature, 1969, 224, 918-919.

Posner, M. I. Chronometric explorations of mind. Hillsdale, N.J: Erlbaum, 1978.

ROBERTs, S. Cross-modal use of an internal clock. Journal of Experimental Psychology: Animal Behavior Processes, 1982, 8, 2-22.

Wilson, W. A., \& Shaffer, O. C. Intermodality transfer of specific discriminations in the monkey. Nature, 1963, $197,107$.

(Received for publication December 23, 1981.) 